Trans

continentales
Transcontinentales

Sociétés, idéologies, système mondial

$12 / 13 \mid 2012$

Marchés de l'art émergents

\title{
Art contemporain arabe
}

Un marché en émergence

Contemporary Arab art. An emerging market

\section{Safia Belmenouar}

\section{(2) OpenEdition}

\section{Journals}

Édition électronique

URL : http://journals.openedition.org/transcontinentales/1317

DOI : 10.4000/transcontinentales. 1317

ISBN : 978-2-7351-1599-0

ISSN : 1775-397X

Éditeur

Editions de la maison des sciences de l'homme

\section{Référence électronique}

Safia Belmenouar, «Art contemporain arabe », Transcontinentales [En ligne], 12/13 | 2012, document 3, mis en ligne le 30 août 2012, consulté le 08 septembre 2020. URL : http://journals.openedition.org/ transcontinentales/1317 ; DOI : https://doi.org/10.4000/transcontinentales.1317

Ce document a été généré automatiquement le 8 septembre 2020

Tous droits réservés 


\title{
Art contemporain arabe
}

\author{
Un marché en émergence \\ Contemporary Arab art. An emerging market
}

Safia Belmenouar

1 L'aspiration démocratique qui souffle sur le monde arabe en offre une nouvelle image. L'état de conflit permanent a eu tendance à évincer la réalité d'une scène artistique qui, malgré l'autoritarisme des régimes et le conservatisme des sociétés, a toujours tenté d'exister. Et cette scène artistique s'inscrit, depuis peu, dans un marché de l'art contemporain en émergence. Comme le souligne le rapport Artprice 2010-2011 sur le marché de l'art, c'est à partir des années 2005-2006 que les artistes arabes contemporains font une entrée plus marquée dans les galeries d'art et les sociétés de ventes aux enchères. La mise en place de ventes spécialisées (Christie's Dubaï, Bonham's Dubaï, Sotheby's Doha) ou la création de foires d'art contemporain à Marrakech et à Dubaï témoignent de cette vitalité. Parallèlement, un certain nombre d'expositions dans les musées et les institutions du monde occidental reflètent une éclosion créative (Unveiled: new art from the Middle East, présentée à la Saatchi Gallery, Londres, 2009 ; The Future of a promise, en parallèle à la 54 Biennale de Venise, 2011). Par ailleurs, les grands travaux muséaux menés sur l'île de Saadiyat d'Abu Dhabi - en partenariat avec le Guggenheim et le Louvre ${ }^{1}$ - sont le signe d'une volonté de pallier le manque de structures culturelles dans la région.

2 Cet article propose d'étudier les modalités d'émergence d'un marché de l'art contemporain arabe dans l'économie internationale de l'art. Cette émergence est à replacer dans un contexte géographique et sociopolitique où la production artistique joue des interdépendances à polarité naissante entre marché régional et marché international. Il conviendra alors d'analyser les interconnexions entre, d'une part, un marché existant en cours de structuration et, d'autre part, un champ culturel en recherche d'homologation et de légitimation internationales. Enfin, cet article éclairera la question de l'intégration des artistes - à partir du parcours de l'un d'entre eux, l'Égyptien Youssef Nabil. En effet, le cas de ce photographe offre un exemple des questionnements relatifs à sa place sur le marché global et aux liens qu'il entretient avec la société d'origine dans laquelle s'ancre son travail esthétique. Ou, autrement dit, 
comment un artiste, originaire des pays dits "périphériques", s'intègre - par sa création et son statut d'artiste - dans le circuit international de l'art, à travers les processus de marchandisation, de consécration, et d'institutionnalisation.

\section{Structuration d'un nouveau marché dans une interconnexion entre local et global}

3 Le développement de comités d'acquisitions spécialisés dans les musées tels que la Tate Modern, le British Museum ou le Guggenheim, a renforcé la présence d'artistes issus de la scène culturelle arabe, jusqu'à il y a peu émergente.Portés par la montée en puissance de l'art contemporain - dont le produit des ventes mondiales aux enchères a décuplé pendant la dernière décennie -, les artistes arabes constituent une manne croissante pour les galeries d'art et les sociétés de ventes aux enchères.

4 Dans son ouvrage À la recherche d'une modernité arabe: l'évolution des arts plastiques en Égypte, au Liban et en Irak, Sylvia Naef rappelle qu'à la fin des années 1980, « un véritable marché de l'art ne s'est pas encore formé dans les pays arabes $\|^{2}$, en raison des conditions sociales et politiques et de l'inexistence d'un public. Jack Persekian, directeur de la Biennale d'art contemporain de Sharjah ${ }^{3}$, souligne, pour sa part, qu'une quinzaine d'années auparavant, l'art de cette région du monde «ne figurait dans aucun des événements artistiques internationaux. Il n'y avait rien - la comparaison doit se faire en remontant dix ans en arrière : de zéro, nous sommes parvenus là où nous en sommes aujourd'hui $»^{4}$. La formation d'un ensemble de structures - institutions, galeries, collectionneurs et, bien entendu, artistes - a permis les conditions d'émergence d'un marché de l'art. Ces structures fonctionnent comme un ensemble d'instances de légitimation - processus d'imbrications complexes de relations à la fois institutionnelles et marchandes - à travers lesquelles les acteurs, en interaction les uns avec les autres, labellisent l'œuvre d'art, au niveau régional et international. L'art contemporain arabe s'inscrit dans cet ensemble. L'impulsion est venue des États du Golfe et a rayonné dans les pays où la création est traditionnellement la plus forte : Égypte, Palestine, Liban, Syrie, Iran.

Depuis 2006, date de l'installation de Christie's à Dubaï, on a donc vu apparaître sur la scène du marché artistique international des artistes de la région qui, jusque-là, n'avaient qu'une notoriété locale. D'autant que l'installation de Christie's à Dubaï sera suivie par celle de Bonham's Dubaï, en 2008, puis celle de Sotheby's Doha, en 2009. À partir des années 2005-2006, les galeries d'art etles sociétés de ventes aux enchères «misaient essentiellement sur la génération d'artistes nés entre 1920 et 1944 pour optimiser leurs recettes» (rapport Artprice 2011) . En effet, «73\% du fruit des enchères pour l'art du Moyen-Orient » (ibid.) était issu de la vente d'œuvres des pères fondateurs de l'art arabe et iranien moderne. Pourtant, en moins de trois années, des jeunes signatures se sont imposées, accroissant un chiffre d'affaires qui est passé d'une « moyenne de $7 \%$ en 2006 à plus de $20 \%$ des recettes en 2008 » (ibid.). Cependant, la crise économique de 2008 a donné un coup d'arrêt à cet essor : une " dégringolade sans précédent » (ibid.) qui a mis en évidence les emballements de la période précédente, ainsi que les pratiques d'un marché trop jeune ${ }^{6}$. Il faudra attendre le début de l'année 2011 pour que le marché de l'art contemporain arabe se stabilise et renoue avec "les niveaux de prix enregistrés fin $2004 »^{7}$. 
Parallèlement à l'installation de maisons de ventes, la tenue de foires internationales d'art contemporain constitue un levier d'action visant à asseoir cette émergence. Les Émirats arabes uniss'affirment comme l'un des nouveaux "eldorados ${ }^{8}$ avec, notamment, la Biennale de Sharjah, créée en 1993 et la foire Art Dubai ${ }^{9}$, créée en 2007. Ces manifestations ont fait de la cité des Émirats la plate-forme marchande de l'art au Moyen-Orient ; les œuvres des différents marchés nationaux - Liban, Syrie, Iran, Égypte - pouvant y être présentées et achetées. Comme le souligne John Martin, cofondateur et directeur de la foire Art Dubai, son but « est d'organiser un salon d'art contemporain de haut niveau au Moyen-Orient, susceptible d'attirer les principales galeries internationales $»^{10}$. Mais, selon un spécialiste de l'Asie chez Christie's, Art Dubai n'attire pas les principaux marchands au niveau international, ce sont plutôt « des œuvres pour des collectionneurs nouveaux, des œuvres, disons d'entrée de gamme $»^{11}$. Cependant, comme l'indique Antonia Carver, nouvelle directrice de la foire Art Dubai, l'édition 2011 marque une rupture par la présence de galeries "pointues intellectuellement ${ }^{12}$, soulignant que «les galeries qui ont atteint un certain niveau international veulent avoir une vision plus large de l'Asie, et elles regardent alors vers Dubaï et Hongkong. Les gens réalisent qu'un déplacement s'est opéré dans le monde de l'art $"^{13}$. Néanmoins, certaines galeries leaders, à l'exemple de Kamel Mennour (Paris) ou Almine Rech (Paris), ont préféré être présentes dans la foire voisine, celle d'Abu Dhabi, dont la dernière édition s'est tenue en novembre 2010. Une certaine compétition demeure donc, même si Antonia Carver semble atténuer cet état de fait : « le modèle à Abu Dhabi est différent, il s'agit d'une foire financée par le gouvernement, alors que nous sommes une entreprise privée. Abu Dhabi veut se créer en centre, alors que Dubaï collabore avec différentes villes du Golfe. Mais, lorsque les musées seront créés à Abu Dhabi, il y aura un effet amplificateur jusqu'à Dubaï. Je crois qu'il y a déjà moins de compétition, et les leaders fédéraux voient Dubaï et Abu Dhabi comme faisant partie d'un même pays uni $»^{14}$.

7 Comme le souligne la sociologue Raymonde Moulin, le marché de l'art de ces trois dernières décennies «ne fonctionne plus comme une juxtaposition de marchés nationaux communiquant plus ou moins bien entre eux, mais comme un marché mondial $»^{15}$. Malgré la concurrence affichée pour l'hégémonie entre les différents marchés des pays du Golfe, le marché de l'art contemporain arabe s'insère parfaitement dans cette redéfinition qui prend la forme d'une « interconnexion de marchés ${ }^{16}$.

\section{Entre marché local limité et recherche de légitimation au niveau global}

8 La réussite économique et financière des États du Golfe a permis la survenue d'une nouvelle catégorie sociale de collectionneurs d'art. Cependant, Sylvia Naef note que, pour un certain public arabe, "aller voir des expositions constituait davantage une activité mondaine que culturelle car, à l'exception de quelques individus, il ne possédait pas de connaissance dans ce domaine $»^{17}$. Une idée que renforce l'analyse de Mohammad Aziza, qui rapporte que«la culture arabe n'a pas développé depensée esthétique. Et d'ailleurs, même si elle en avait une, elle n'aurait pas eu de poids face aux normes européennes $»^{18}$. Chantal Crousel, qui possède une galerie à Paris, va dans le même sens : "Il s'agit d'une région sans critique d'art, sans musée, sans commissaire d'exposition formé, mais où il y a beaucoup de consultants, d'argent et de maisons de 
ventes. Ce que nous pouvons apporter prendra du temps, avec d'autres exemples d'art et de façons de collectionner. Cela prendra une petite génération ${ }^{19}$. Pourtant, il faut rappeler, comme l'indique Raymonde Moulin, qu' «à l'occasion des premières ventes, près de $60 \%$ des acquéreurs étaient originaires du Moyen-Orient, les plus fortes enchères étant obtenues par des artistes arabes, égyptiens et iraniens ${ }^{20}$.Ces collectionneurs sont à l'origine d'un élargissement du marché en termes d'augmentation du volume financier. Toutefois, on peut se poser la question de leurs motivations : en agissant sur le marché, ils s'approprient un art en provenance de leur région, mais fondent-ils une réelle "possibilité d'existence des objets d'art et, en conséquence, celles des artistes ${ }^{21}$ ?En effet, en l'absence d'institutions d'arbitrage telles que musées, critiques et historiens d'art, indépendants des enjeux marchands, ne risque-t-on pas « d'aboutir à une création commanditée et contrôlée par une poignée

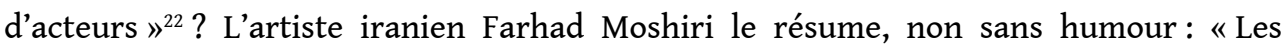
intellectuels se sont transformés en commissaires, les femmes au foyer en peintres et les mondaines en marchands d'art et en galeristes. Maintenant que le marché s'est retourné, ces professions nouvellement créées reviennent résolument à ce qu'elles étaient. Seuls ceux qui sont sincères et vraiment intéressés par ce qui se passe continuent à travailler, à créer, produire et acquérir le meilleur $»^{23}$.

Cet engouement pour l'art arabe contemporain se traduit, notamment au niveau international, à travers une présence accrue lors de manifestations et d'expositions. La $54^{e}$ édition de la Biennale de Venise ${ }^{24}$ n'avait jamais accueilli un aussi grand nombre d'artistes de cette région du monde. L'exposition The Future of a promise - en parallèle à la Biennale de Venise et produite par Edge of Arabia - a compté vingt-deux artistes du monde arabe parmi lesquels, Mona Hatoum (Liban), Kader Attia (Algérie) et Mounir Fatmi (Maroc) ; d'autant mieux connus que la plupart d'entre eux résident en Europe ou aux États-Unis, contrairement à d'autres qui travaillent au Liban, en Palestine ou dans les pays du Golfe. La commissaire de l'exposition, Lina Lazaar, a pris en compte un postulat commun à tous: la mise en forme visuelle du politique, ignorant ainsi les différences de notoriété ou de situation géographique. Installations, photos, peintures : « les artistes arabes se saisissent de tous les modes d'expression de l'art actuel, simples ou techniques, figuratifs ou allégoriques $\aleph^{25}$. Mondialisées, ces pratiques artistiques sont désormais largement répandues et elles ont précédé ce qui est communément appelé «le printemps arabe». Les vecteurs de communication - Internet, réseaux sociaux, vidéo sur téléphone portable - ont certainement rendu ces modes d'expression plastique plus familiers.

10 Aussi, le rapport entre le "printemps arabe » et la présence d'artistes arabes à la Biennale de Venise est-il un rapport de corrélation et, sans doute, de connivence. Pour autant, seul le pavillon égyptien y fait référence. Il est dédié au vidéaste et performer Ahmed Basiouny, tué par balles sur la place Tahrir, le 28 janvier 2011. Sa dernière œuvre - une performance dans la ville du Caire, réalisée en 2010 - y est projetée, suivie des dernières images qu'il a filmées, la veille de son assassinat. Pour d'autres pays tels que les Émirats arabes unis, l'Arabie saoudite, l'Iran, pas de référence politique mais plutôt «la conviction que les pays en question cherchent à exister sur la scène artistique $»^{26}$. 


\section{Youssef Nabil, un art photographique globalisé ?}

11 En Égypte, l'art contemporain connaît, depuis le début des années 1990, quelques frémissements avec l'installation de quelques galeries cairotes (TownHouse Gallery, Mashrabia) qui se sont développées grâce à des financements privés et à leurs connexions avec des fondations et des festivals internationaux ${ }^{27}$. Offrant les premiers espaces de monstration, ces initiatives ne parviennent pourtant pas à remédier à une situation relevant, jusqu'au milieu des années 2000, pour l'essentiel d'un «sousdéveloppement ». La non-existence d'un «monde de l'art» au niveau local concourt à l'isolement des artistes dans un environnement peu dynamique. Comme le souligne Nicolas Bourriaud, "rares sont les artistes provenant des pays "périphériques" ayant réussi à intégrer le système central de l'art contemporain en continuant à résider dans leur pays d'origine $»^{28}$. Malgré l'émergence d'un marché local ou la constitution de galeries locales, «ceux qui accèdent à ce type de carrière internationale ont été pris en charge par une grande galerie, nord-américaine ou européenne $»^{29}$.

12 C'est le cas de Youssef Nabil, né au Caire en 1972, passé maître dans l'art du portrait et dont le travail est notamment représenté par la galerie Nathalie Obadia (Paris) et la Yossi Milo Gallery (New York). Récompensé en 2003, par le prix Seydou Keita décerné lors de la Biennale de photographie de Bamako (Mali), Youssef Nabil a participé à nombre d'expositions : la Villa Medici à Rome, la Biennale de Venise, les Rencontres internationales de la photographie d'Arles, le Kennedy Center à Washington, l'Institut du monde arabe à Paris, Aperture Foundation de New York, le British Museum de Londres, Centro de la Imagen à Mexico... Par ailleurs, ses œuvres font déjà partie de collections d'institutions de la scène internationale comme celles de la Maison européenne de la photographie (Paris), la Fondation Louis Vuitton (Paris), le Victoria \& Albert Museum (Londres), le Mathaf - musée d'Art moderne arabe - (Doha) ou la collection Fayam (Dubaï). Une première rétrospective lui a d'ailleurs été consacrée à la Maison européenne de la photographie en $2012^{30}$. Enfin, son travail a donné lieu à la publication de deux monographies : Sleep in My Arms ${ }^{31}$ et I Won't Let You Die ${ }^{32}$.

13 À l'instar d'autres jeunes signatures, l'artiste égyptien, depuis son introduction aux enchères en 2007, affiche de bons résultats. Utilisant la photographie, médium particulièrement prisé par cette jeune génération, ses œuvres sont adjugées en moyenne entre 5000 et 50000 euros. Ainsi, la demande a fait doubler le prix de certains clichés en un temps très court. La photographie Maelema-Fifi Abdo au narguilé en est un exemple. En novembre 2008, l'un des trois tirages était vendu 19000 euros chez Bonham's Dubaï. Deux ans plus tard, en octobre 2010, l'exemplaire $1 / 3$ sera vendu 52800 euros chez Christie's Dubai ${ }^{33}$. Le prix de l'œuvre aura pris plus de $179 \%$. Et l'exemple se répète. La photographie Ehsan \& Amp/Light, Cairo (exemplaire 3/3) était vendue «autour de $32000 €$ en octobre 2010 (Sotheby's Londres) avant d'atteindre $41000 €$, six mois plus tard (Christie's Dubaï, 19 avril 2011) $»^{34}$. Le travail de Youssef Nabil bénéficie d'une double conjoncture favorable. En effet, l'artiste est à la fois porté par l'engouement autour de l'art du monde arabe dû à la présence de nouvelles signatures et par l'émergence d'un marché de la photographie contemporaine, structuré et en pleine expansion.

14 Dès lors, sa réputation est autant le résultat d'un parcours artistique au sein des différentes instances de légitimation que la manifestation d'indices - exposition, achat muséal, ventes aux enchères, monographies, collections privées, etc. - qui attestent de 
la reconnaissance de son travail, et donc de sa place sur le marché.La valeur de l'art, et dans ce cas, la valeur de l'œuvre de Youssef Nabil, se constitue à l'articulation du marché et du musée. Et, Alain Quemin, de préciser: «dans un monde qui recherche sans cesse des signes de la valeur esthétique et financière des œuvres, la nationalité de leurs auteurs semble constituer un critère, notamment dans le cadre de stratégies de minimisation du risque associé au choix des artistes et de leurs œuvres $»^{35}$.

Les modèles photographiques de Youssef Nabil sont des personnalités contemporaines, issues du paysage culturel, tels les artistes Shirin Neshat, Ghada Amer, Marina Abramovic, Gilbert \& Georges, la chanteuse Natacha Atlas, l'architecte Zaha Hadid ou encore les actrices Rossy de Palma et Catherine Deneuve. La technique mise en œuvre par l'artiste est toujours la même: ce sont des images argentiques, noir et blanc, colorisées à la main avec de l'aquarelle, parfois mélangée à de l'huile et rehaussée de crayon. Une technique issue de l'artisanat égyptien qui donne aux clichés ce côté suranné et fané. Le photographe reconnaît l'influence du cinéma égyptien des années 1950, des affiches de cinéma de la même époque, ainsi que des portraits de famille en noir et blanc colorisés. Auprès d'artisans coloristes, Youssef Nabil s'initie à cette technique en vigueur dans les studios du Caire et d'Alexandrie d'autrefois. Inspirés d'une culture cinématographique disparue, ces tirages sont des hommages rendus aux artistes d'hier. Retouchés au pinceau, ils aspirent à imiter le grain de la peau en accentuant les contours des visages, des yeux et des lèvres, le plissé des costumes, l'aspect poudreux ou vaporeux de la toile de fond devant laquelle pose le modèle. Ces effets techniques produisent une impression d'étrangeté et d'irréalité. François Soulages, dans son Esthétique de la photographie, la perte et le reste, rappelle que la photographie «est toujours faite par un homme qui est lui-même travaillé et dominé inconsciemment par des modèles à reproduire ou à éviter, par des pulsions et des désirs $»^{36}$. Les portraits de Youssef Nabil dialoguent, non sans nostalgie, sur un mode de narration complexe, avec un temps passé. D'autres portraits défient les discours et les clichés tenus sur le monde arabe ou sur les artistes photographiés: l'artiste Ghada Amer, connue pour ses travaux sur un mode pornographique, apparaît voilée ; Natacha Atlas pose à la manière d'une odalisque, "fumeuse au narguilé ». En s'attachant à remettre en cause les clichés, le photographe crée de nouvelles mythologies autour d'un Orient rêvé. Ou encore, il caricature l'Orient réduit à la seule image d'un fanatisme religieux. La mise en scène et le travail « d'après-coup " placent ces visages dans une "nouvelle théâtralité $»^{37}$, prouvant une nouvelle fois que «toute photographie est "théâtralisante" " ${ }^{38}$.

Dans un dialogue fécond avec l'artiste iranienne Shirin Neshat, Youssef Nabil s'interroge sur sa place dans un monde de l'art mondialisé. Selon les deux artistes, un risque demeure quant à une forme "d'orientalisation de leur culture " ${ }^{39}$.Shirin Neshat l'exprime ainsi:«Bien que nous soyons du Moyen-Orient et que nos sujets fassent souvent référence à notre propre culture, notre travail est vu par des Occidentaux. [...] Il semble qu'il faille nous battre des deux côtés. D'un côté, en Occident, nous résistons aux clichés occidentaux et aux stéréotypes véhiculés sur notre culture, en essayant d'insister sur la complexité de nos sociétés aussi bien que sur la valeur universelle de notre art. De l'autre, au Moyen-Orient, nous faisons souvent face aux préjugés car on nous suspecte en tant qu'artistes ne vivant plus dans leur propre pays, mais qui choisissent des sujets liés à leur culture d'origine $»^{40}$. 
17 L'artiste questionne ici le fait du multiculturalisme, remis en cause par Nicolas Bourriaud dans son ouvrage Radicant, et qui s'avère avant tout politique: "l'art contemporain s'accorde progressivement au mouvement de la globalisation, qui standardise les structures économiques et financières tout en faisant de la diversité des formes le reflet inversé, mais exact, de cette uniformité " ${ }^{41}$. Et d'ajouter : " dans l'espace idéologique "multiculturel", un bon artiste se doit ainsi de témoigner de son "identité culturelle", comme si, il ou elle, portait un tatouage indélébile » (p. 192). Comme en témoignent Youssef Nabil ou Shirin Neshat, ces derniers craignent d'être prisonniers de cette « différence » culturelle qui, à la fois, les valorise sur le marché international mais risque de les assigner à une identité culturelle. Ces artistes originaires des pays dits de la "périphérie " réussissent à traiter les signes de leur culture d'origine depuis un « centre » qui les a consacrés et qu'ils ont, généralement, rejoint. D’un point de vue économique, esthétique et institutionnel, les pôles dominants de la sphère du monde de l'art qui «labellisent» restent «aujourd'hui encore et en dépit des dénégations, toujours contrôlés par le seul monde occidental ». Nicolas Bourriaud appelle néanmoins à une alternative à cet état de fait qu'il nomme l'interculturalisme. Basé sur un « double dialogue » (p. 193), celui-ci s'articule autour de la relation que l'artiste entretient avec sa tradition culturelle, tout en s'inscrivant dans l'ensemble des valeurs économiques et esthétiques qui fonde le débat sur « la mondialité » artistique et son mode d'existence. Youssef Nabil serait alors un exemple de ces « artistes interculturalistes » qui, par des «actes de réenracinements successifs» (p.189), traduisent un jeu complexe de "résonances et résistances" (p.190) entre leur culture d'origine revisitée, réinterprétée et les destinataires de leurs œuvres (le public occidental).Autrement dit, se joue une contemporanéité esthétique et culturelle usant de référentiels à la fois locaux et globaux.

\section{En conclusion}

18 L'économie internationale de l'art s'est redessinée, ces dernières années, en incorporant des marchés, jusqu'à présent, locaux, à l'exemple du marché de l'art contemporain arabe. Cette nécessité d'extension répond à un besoin d'offre sans cesse renouvelée, afin d'approvisionner en œuvres d'art un marché au volume financier en constante augmentation.

19 Néanmoins, en restant sous le contrôle d'un «main stream occidental » ${ }^{42}$, cette ouverture vers de nouveaux marchés montre un certain nombre de limites. En effet, " les acteurs culturels et économiques chargés de découvrir, sélectionner et valoriser les artistes et les œuvres d'art $»^{43}$ proviennent souvent d'un même monde, celui de l'art occidental. Le jeu d'interdépendances entre marchés régional et international reste donc soumis à des interconnexions qui, par leur structuration, se définissent par un mode de fonctionnement formel ou informel préétabli. Ainsi, pour les artistes en provenance des pays dits " périphériques », l'une des difficultés réside dans la tentative d'exister sur une scène internationale, en dehors d'un centre hégémonique contrôlant l'élaboration des valeurs économiques et esthétiques. Alain Quemin rappelle que «tant le marché que la consécration institutionnelle restent aux mains de pays occidentaux, en particulier des plus riches d'entre eux $»^{44}$. La création de foires, l'installation de sociétés de ventes aux enchères ou de galeries contribuent à la marchandisation d'œuvres validées notamment par le «cosmopolitisme des commissaires 
d'expositions $»^{45}$ ou par l'organisation en réseaux de musées ou d'institutions d'accueil des artistes. L'émergence d'un marché de l'art contemporain arabe se produit donc comme une réplique de mécanismes de valorisation, c'est-à-dire «des signes de la valeur esthétique et financière des œuvres ${ }^{46}$, déjà éprouvés.

Cependant, alors qu'il y a une dizaine d'années, ces lieux de visibilités locales ne concurrençaient pas ceux organisés dans le monde occidental, cet état de fait semble avoir quelque peu évolué. Alain Quemin constate que «le marché n'est nullement abandonné aux mains de rivaux potentiels $\aleph^{47}$ et reste fortement concentré en Europe et aux États-Unis, ces derniers comptant « l'essentiel des ventes aux enchères, les plus grandes foires et les galeries les plus influentes $»^{48}$; cependant, les pays dits "périphériques" n'en sont plus aujourd'hui totalement absents. Du côté de l'Asie, provient la plus forte impulsion puisque, désormais, cinq capitales asiatiques sont classées parmi les dix premières places de marchés aux enchères d'art contemporain Pékin étant, derrière New York, la deuxième place pour la vente d'œuvres contemporaines. Du côté du monde arabe, Dubaï consolide sa position de plate-forme marchande par sa présence au sein de ce classement à la dixième position ${ }^{49}$.

Par ailleurs, se pose également la question des conditions d'émergence d'une production artistique en provenance du monde arabe qui, comme le remarque Raymonde Moulin, participe à "une homogénéisation artistique mondiale sous le contrôle d'un pôle dominant politique, économique et artistique " ${ }^{50}$. L'un des signes de cette homogénéisation étant "celui des supports utilisés par les artistes de la périphérie qui se servent de photos, vidéos, installations comme d'un "passeport" pour entrer dans le champ artistique et le marché occidental ». Relayant ce questionnement, on peut se demander alors en quoi l'utilisation de ces supports ne devrait être que le fait d'artistes dits du « centre ». Pourquoi, comme elle l'écrit, « dans cette perspective, la circulation [...] des modèles occidentaux serait la [...] négation de la diversité culturelle ». Car, ne s'agit-il pas avant tout, à l'exemple de Youssef Nabil, de la capacité ou de la compétence de chaque artiste qui accède à un statut international, de «jouer des frontières géographiques »? Ces "artistes interculturalistes » dont la production artistique est le fruit de métissages et d'hybridations, enrichissent des « dynamiques de différenciation ».

\section{NOTES}

1. Est également en projet un centre de concert et de performance. En 2008, s'est ouvert un musée d'Art islamique au Qatar.

2. Paris, Slatkine, $1996: 327$.

3. Principauté arabe voisine de Dubaï.

4. Sabine Le Stum, «Moyen-Orient, moins d'argent, plus de temps », Artpress, $n^{\circ} 361$, novembre 2009, Paris : 52 .

5. 5 «Le marché de l'art contemporain 2010/2011, le rapport annuel Artprice » [en ligne], Artprice, 2011: 41, consulté le 24 juillet 2012. URL: http://imgpublic.artprice.com/pdf/ fiac11fr.pdf 
6. «La moitié des œuvres soumises à enchères furent ravalées au $2^{\mathrm{e}}$ semestre 2008 , puis l'indice des prix chutait de $-42 \%$ sur la seule année 2009. » À l'automne 2008, Christie's a connu un revers brutal avec $70 \%$ des lots vendus à $65 \%$ de leur estimation. La vente de 2009 a réalisé $85 \%$ de son estimation, mais avec un chiffre d'affaires de 4,7 millions de dollars, presque deux fois moins que sa première vente de mai 2006. Source Artprice.

7. Ibid.

8. Raymonde Moulin, Le marché de l'art. Mondialisation et nouvelles technologies, Paris, Flammarion, 2009 : 77.

9. Notons qu'Art Dubai est financé par des capitaux privés : son principal sponsor est Abraaj Capital, une société de capital-risque qui a pour investisseurs les familles royales de la région.

10. «Art Dubai : entretien avec John Martin. Cofondateur et directeur d'Art Dubai », One Fine Art, $\mathrm{n}^{\circ} 318$, mars $2008: 31$.

11. Judith Benhamou-Huet, « Dubaï confirme sa place artistique », Les Échos, 19 mars 2010.

12. Comme par exemple, The Hotel (Londres), Balice Hertling (Paris) ou Johann König (Berlin).

13. "Dubaï reste attractif. Sur fond de crise, Art Dubai rassemble des galeries internationales très pointues ", Le Journal des Arts, $\mathrm{n}^{\circ}$ 342, 4 mars 2011.

14. Ibid.

15. Raymonde Moulin, op. cit. : 63.

16. Ibid.

17. Sylvia Naef, À la recherche d'une modernité arabe : l'évolution des arts plastiques en Égypte, au Liban et en Irak, Paris, Slatkine, $1996: 327$.

18. Citée par Sylvia Naef, op. cit. : 333.

19. «Dubaï reste attractif. Sur fond de crise, Art Dubai rassemble des galeries internationales très pointues », Le Journal des Arts, $\mathrm{n}^{\circ}$ 342, 4 mars 2011.

20. Raymonde Moulin, op. cit. : 77-78.

21. Michel Melot, «La notion d'originalité et son importance dans la définition des œuvres d'art », in Raymonde Moulin (dir.), Sociologie de l'art, Paris, La Documentation française, 1986 : 201. 22. Victoria Ambrosini-Chenivesse, «Dubaï et les avatars régionaux du boum de l'art », Artpress, $\mathrm{n}^{\circ} 341$, janvier 2008, Paris : 36 .

23. Sabine Le Stum, art. cité : 54 .

24. Du 4 juin au 27 novembre 2011.

25. Philippe Dagen, «Venise galvanisée par le "printemps arabe” et l'Orient », Le Monde, mardi 7 juin 2011.

26. Ibid.

27. «Au cours des deux dernières années, de nouveaux espaces ont ouvert en Égypte : le CIC (Contemporary Image Collective), regroupement de quelques photographes ayant pour objectif de soutenir le développement de la photographie au Caire, et l'ACAF (Alexandria Contemporary Art Forum), qui tente de créer une nouvelle dynamique artistique à Alexandrie et a lancé un programme d'atelier d'art sur le net, pour la vidéo et les films d'animations. » Victoria Ambrosini-Chenivesse, "Art, kitsch et nationalisme en Égypte et en Syrie », Artpress, n³33, janvier 2001, Paris : 56.

28. Nicolas Bourriaud, Radicant, Paris, Denoël, $2009: 189$.

29. Raymonde Moulin,op. cit. : 78.

30. Exposition du 18 janvier au 25 mars.

31. Autograph ABP and Michael Stevenson, 2007.

32. Hatje Cantz, 2008.

33. Source : «Le marché de l'art contemporain 2010/2011, le rapport annuel Artprice », rapport cité.

34. Ibid. : 46. 
35. «Le rôle des pays prescripteurs sur le marché et dans le monde de l'art contemporain » [en ligne], Direction générale de la coopération internationale et du développement, rapport du ministère des Affaires étrangères, juin 2011: 114, consulté le 25 juillet 2012. URL: http:// www.diplomatie.gouv.fr/fr/lMG/pdf/

Le_role_des_pays_prescripteurs_sur_le_marche_et_dans_le_monde_de_1_art_contemporain.pdf

36. Paris, Nathan, $1998: 64$.

37. Ibid. : 65 .

38. Ibid.

39. "Youssef Nabil in conversation with Shirin Neshat ", entretien [en ligne], New York, juin 2008, consulté le 25 juillet 2012. URL: http://www.youssefnabil.com/articles/ in_conversation_with_shirin_neshat.html

40. Ibid.

41. Nicolas Bourriaud, op. cit. : 190. Les citations qui suivent sont du même ouvrage.

42. Raymonde Moulin,op. cit. : 78.

43. Ibid.

44. Alain Quemin, "L'illusion de l'abolition des frontières dans le monde de l'art contemporain international : la place des pays "périphériques" à "l'ère de la globalisation et du métissage" ", Sociologie et sociétés, vol. 34, $\mathrm{n}^{\circ} 2,2002$.

45. Raymonde Moulin,op. cit. : 78.

46. Alain Quemin, art. cité.

47. "Le rôle des pays prescripteurs sur le marché et dans le monde de l'art contemporain ", rapport cité, juin $2011: 115$.

48. Ibid.

49. Source : «Le marché de l'art contemporain 2010/2011, le rapport annuel Artprice », rapport cité.

50. Raymonde Moulin,op. cit. : 79.Les citations qui suivent sont du même ouvrage.

\section{RÉSUMÉS}

Encore considérée comme émergente, la scène artistique en provenance du monde arabe a précédé ce qui est communément appelé «le printemps arabe ». En effet, c'est à partir des années 2005-2006 que les artistes arabes contemporains ont fait une entrée plus marquée dans un marché de l'art en voie de globalisation. Un certain nombre de pays dits " périphériques » sont ainsi intégrés à une scène artistique internationale redessinée, participant de ce fait à sa mondialisation. Au niveau régional, un certain nombre d'initiatives impulsées par les États du Golfe accompagnent cette émergence, notamment en termes d'offre culturelle et marchande travaux muséaux menés sur l'île de Saadiyat, création de foires d'art contemporain à Dubaï et Abu Dhabi, installation des maisons de ventes Christie's ou Sotheby's. Ces leviers d'action sont autant de signes visant à asseoir cette émergence afin que les œuvres d'artistes nationaux (Liban, Syrie, Égypte, Iran...) puissent enfin accéder à un marché local, voire international. Replacé dans un contexte géopolitique à polarité naissante, cet article se propose d'analyser la situation de cette production artistique issue du monde arabe, par rapport à la problématique de son entrée dans l'économie internationale de l'art. Et cela, au regard d'un processus de marchandisation et d'institutionnalisation qui structure un jeu d'interconnexions entre marché local et marché 
international. La question de l'intégration des artistes dans un champ culturel, en recherche d'homologation et de légitimation, éclairera les interrogations de certains d'entre eux, entre crainte d'« orientalisation de leur culture » et récupération «multiculturelle » par le monde de l'art contemporain. À cet égard, l'artiste égyptien Youssef Nabil offre une production jouant de référentiels esthétiques à la fois locaux et globaux.

Still considered as emerging, the Arabic artistic scene did in fact precede what we refer to as the Jasmin revolution. The Arab artists made a noticed breackthrew in 2005-2006 on the globalizing art market. A certain number of countries, previously thought of as peripheral, where then taken in to account as part of a redesigned international artistic scene, hence making it part of the globalization. At a regional level this phenomenon is accompanied by a certain number of initiatives nurished by the Gulf States, both cultural and commercial initiatives are set forward museum construction on the Island of Saadiyat, creation of the contemporary art fairs held in Dubai and in Abu Dhabi, opening of the auction houses Sotheby's and Christie's. These actions are all signs giving weight to an upcoming Arab artistic scene where artworks of national artistes (Libanon, Syria, Egypte, Iran) will finally be aloud access to a local, or even international, market. Placed in a geopolitical context with an appearing polarity, this article is offering to analyse the situation of the artistic production emerging from the Arab world, setting it in relation to the difficulties of merging it into the international art market. And this keeping in mind the process of merchandising and institutionalising structuring a board of connexions between the local market and the international market. The question of the integration of the artist on a cultural level, in search of approval and legitimacy, will illuminate the questions that certain of them have beetween the fear of "orientalisation of their culture" and the "multicultural" appropriation by the contemporary art world. In this sense, the artist Youssef Nabil, offers a production playing both with local as well as global aesthetic references.

\section{INDEX}

Keywords : contemporary arab artists, art market, globalization, photography.

Mots-clés : artistes arabes contemporains, marché de l'art, mondialisation, photographie, émergence

\section{AUTEUR}

\section{SAFIA BELMENOUAR}

Doctorante à l'université Paris-VIII Saint-Denis, Laboratoire EA 4010, AIAC « arts des images et art contemporain » ufrarts@univ-paris8.fr 